Relations industrielles

Industrial Relations

\title{
A Practical Guide to Flexible Working Hours, par Stephen Baum et W. McEwan Young, New Jersey, Noyes Data Corporation, 1974, 190 pp.
}

\section{Laurent Bélanger}

Volume 29, numéro 2, 1974

URI : https://id.erudit.org/iderudit/028515ar

DOI : https://doi.org/10.7202/028515ar

Aller au sommaire du numéro

\section{Éditeur(s)}

Département des relations industrielles de l'Université Laval

\section{ISSN}

0034-379X (imprimé)

1703-8138 (numérique)

Découvrir la revue

Citer ce compte rendu

Bélanger, L. (1974). Compte rendu de [A Practical Guide to Flexible Working Hours, par Stephen Baum et W. McEwan Young, New Jersey, Noyes Data Corporation, 1974, 190 pp.] Relations industrielles / Industrial Relations, 29(2), 409-410. https://doi.org/10.7202/028515ar

Tous droits réservés (C) Département des relations industrielles de l'Université Laval, 1974
Ce document est protégé par la loi sur le droit d'auteur. L’utilisation des services d'Érudit (y compris la reproduction) est assujettie à sa politique d'utilisation que vous pouvez consulter en ligne.

https://apropos.erudit.org/fr/usagers/politique-dutilisation/ 
retrouver pour la plupart à poser les mêmes gestes au même moment: c'est le phénomène de l'encombrement. A titre d'exemples la bousculade pour attrapper le métro ou l'autobus, les embouteillages aux heures d'entrées et de sorties des bureaux, l'inaccessibilité aux terrains de camping durant le mois d'août, en France. Tous ces inconvénients dont la gravité s'accentue de jour en jour nous amènent à repenser l'utilisation du temps pour en effectuer de nouveaux découpages de même qu'à l'aménagement des espaces, puisque temps et espace vont de pair et que ce n'est pas uniquement par un aménagement du temps qu'on va régler le probème de la rareté relative de l'espace.

Dans les sociétés fortement industrialisées axées sur le «plus-être » collectif, on a appris à allouer et à découper le temps en fonction de critères économiques. "Time is Money 》 pour reprendre une expression de nos voisins, les Américains. Il faudrait arrêter de considérer le temps uniquement comme une ressource, une durée, une quantité. On découvrirait ainsi la valeur du moment, celui qui fait qu'une activité est plus intéressante parce qu'on a choisi le moment pour s'y adonner. Evidemment, je veux bien admettre que ce n'est pas possible pour tout le monde; cependant, ce nest pas une raison pour demeurer indifférent, puisque imaginer de nouvelles façons d'aménager son temps est déjà un début vers l'amélioration de la vie de travail et hors-travail.

C'est un peu le genre de réflexions que fait l'auteur et que je reprends dans mes propres termes. Mais, Jacques De Chalendar dépasse l'analyse du phénomène de l'encombrement pour élaborer des mécanismes qui permettraient de l'éliminer en partie. Il décrit des formes déjà connues et des formes récentes d'aménagement du temps; telles que le décalage des horaires fixes, les horaires personnalisés, les horaires flexibles. Il rappelle des expériences récentes telle que la semaine comprimée, l'étalement des congés au niveau de la semaine de travail.

Le phénomène des vacances annuelles retient aussi son attention. Il suggère d'en repenser la répartition par une division de l'année en deux parties, deux saisons: une période de pleine activité intense tant dans le secteur de l'écono- mie que celui de l'éducation entre les mois d'octobre et d'avril ; une période d'activité réduite de mai à septembre pendant laquelle une proportion de $25 \%$ à $30 \%$ de la population active serait en congé. A l'échelle de la vie de travail, il reprend des mesures déjà connues en particulier, l'abaissement de l'âge de la retraite obligatoire, la réglementation concernant les congés sabbatiques et l'entrée sur le marché du travail. L'ouvrage comprend aussi une annexe qui traite du budget-temps, du travail à temps partiel, de l'utilisation des temps libres. En conclusion, l'auteur fait un retour historique sur les notions de travail et de loisir pour se demander si la société de demain permettra un rapprochement de ces deux notions après les avoir pendant longtemps considérées comme opposées.

Sans chercher à effectuer une analyse systématique et approfondie des phénomènes qui retiennent son attention, Jacques De Chalendar réussit quand même à susciter le réflexion sur un sujet qui prend de plus en plus d'importance : celui de l'aménagement du temps. Cette réflexion doit se poursuivre puisqu'elle contient des possibilités d'amélioration du genre de vie qu'offre la société postindustrielle dans l'état actuel de son développement.

Université Laval

Laurent BELANGER

\section{A Practical Guide to Flexible Working} Hours, par Stephen Baum et W. McEwan Young, New Jersey, Noyes Data Corporation, 1974, 190 pp.

A ma connaissance, c'est un des premiers ouvrages publiés en anglais sur les horaires flexibles. Il couvre assez bien le sujet mais sous un angle très technique. L'auteur démarre par une réflexion sur la notion du travail à travers les âges et les nouvelles aspirations des travailleurs. Il situe les horaires flexibles parmi les nouvelles tentatives qui visent à redonner aux individus une certaine possibilité de choix dans l'organisation de leur travail, en particulier, l'aménagement des temps de travail. Les auteurs abordent ensuite les principes de base qui soustendent ce nouveau système d'horaire; les avantages et les inconvénients du point de vue de l'entreprise et de celui des diverses catégories de personnel. 
L'étude des différents systèmes de report et de décompte des heures effectivement travaillées et des problèmes spécifiques reliés à l'introduction du système constitue l'objet des quatrième et cinquième chapitres.

Les sixième et septième chapitres présentent de façon détaillée les principes d'implantation du système et un modèle exploratoire qui pourrait recevoir multiples adaptations respectant les caractéristiques propres à chaque entreprise; caractéristiques du marché, de la technologie utilisée; de la nature des tâches. Ceci constitue, à mon avis, la contribution spécifique des deux auteurs de cette publication puisqu'on ne retrouve pas dans les ouvrages français un exposé aussi clair et méthodique des principes, des procédures et des mécanismes d'implantation des horaires flexibles. Un dernier chapitre qui traite des attitudes syndicales à l'endroit de ce système m'apparaît le plus faible. De fait, même si les auteurs ont procédé à une enquête rapide auprès de quelques centrales syndicales, on peut difficilement, à la lumière des résultats, se faire une idée exacte de ce que pensent les syndicats sur le sujet. Il serait peut-être plus juste de conclure que les centrales visitées n'ont pas de positions bien articulées sur le sujet. Au Canada et aux Etats-Unis, la situation m'apparaît la même pour le moment. Je n'ai pu, à travers les ouvrages que j'ai consultés au cours des derniers mois retrouver une prise de position syndicale bien précise sur cette forme récente d'aménagement du temps de travail. C'est donc un sujet qui se prêtera bientôt à une recherche plus approfondie.

Dans l'ensemble, le volume excelle par son caractère technique et toute personne préposée à l'implantation des horaires flexibles trouverait avantage à le consulter.

\section{Université Laval}

Laurent BELANGER

The Brandon Packers Strike, by G.F. Macdowell, Toronto / Montréal, McClelland and Stewart Limited, 1971, $305 \mathrm{pp}$.

1960. Une petite ville dans une région rurale. Un abattoir. Un syndicat et, un beau jour, une grève qui fit parler d'elle longtemps au pays manitobain.
La petite ville, c'est Brandon. A l'époque, moins de trente mille habitants, métropole d'une immense zone agricole qu'elle approvisionne et dont elle transforme les produits; c'est aussi un centre ferroviaire assez important. Elle avait été jadis une petite cité ambitieuse qui avait même aspiré à supplanter la toutepuissante Winnipeg. Cela n'eut pas de suite. Malgré les efforts de ses dirigeants et de ses hommes d'affaires, elle était restée jusque-là sous-développée.

L'entreprise. Elle s'appelait Brandon Packers. Avec ses 110 employés, elle était quand même l'entreprise principale de la ville. Elle avait vu le jour en 1936. Le propriétaire, fils d'un boucher qui avait réussi, après avoir travaillé à gauche et à droite et acquis ainsi un bagage raisonnable d'expérience pratique, revint s'établir dans sa petite ville natale où, graduellement, il transforma l'état paternel et un véritable abattoir. Commencée avec un investissement de vingt mille dollars, l'usine qui comptait sept employés au départ, en groupait cent cinquante, cinq années plus tard. C'était alors la guerre et la demande ne faisait plus problème. Ce qui importait d'abord, c'était d'accroître rapidement la capacité de production pour répondre à des besoins immédiats et urgents. Comme le bacon et le jambon étaient fort en demande, l'usine se spécialisa dans l'abattage et la transformation des produits de porc. La fin de la guerre obligea à certains ajustements, mais l'entreprise continuait à être rentable. Cependant, au milieu de la décennie 1950, un changement majeur devait se produire. Le propriétaire vieillissait. De plus, il s'était laissé tenter par la politique et, conséquence, il négligeait un peu l'administration de son affaire qui n'en conservait pas moins une excellente réputation de solvabilité, en particulier auprès des fermiers des localités environnantes.

En 1956, il met son entreprise en vente entre les mains d'un agent immobilier de Winnipeg. Deux types de Toronto s'en portent acquéreurs, l'ancien propriétaire, gardant une partie substantielle des parts de la société. Le changement de propriétaire n'entraîna pas immédiatement un changement de direction. $\mathrm{Ce}$ ne fut qu'en 1958 qu'un nouveau gérant allait être nommé.

Un syndicat. C'était une section locale des United Packinghause Workers of 